\title{
Mining metagenomic whole genome sequences revealed subdominant but constant Lactobacillus population in the human gut microbiota
}

\author{
Maddalena Rossi, ${ }^{*} \dagger$ Daniel Martínez-Martínez, ${ }^{2,3,4 \dagger}$ \\ Alberto Amaretti, ${ }^{1}$ Alessandro Ulrici, ${ }^{1}$ \\ Stefano Raimondi ${ }^{1}$ and Andrés Moya ${ }^{2,3,4}$ \\ ${ }^{1}$ Department of Life Sciences, University of Modena \\ and Reggio Emilia, Modena, Italy. \\ ${ }^{2}$ Cavanilles Institute of Biodiversity and Evolutionary \\ Biology, University of Valencia, Valencia, Spain. \\ ${ }^{3}$ Foundation for the Promotion of Health and Biomedical \\ Research in the Valencian Community (FISABIO), \\ Valencia, Spain. \\ ${ }^{4}$ Network Research Center for Epidemiology and Public \\ Health (CIBER-ESP), Madrid, Spain.
}

\section{Summary}

The genus Lactobacillus includes over 215 species that colonize plants, foods, sewage and the gastrointestinal tract (GIT) of humans and animals. In the GIT, Lactobacillus population can be made by true inhabitants or by bacteria occasionally ingested with fermented or spoiled foods, or with probiotics. This study longitudinally surveyed Lactobacillus species and strains in the feces of a healthy subject through whole genome sequencing (WGS) data-mining, in order to identify members of the permanent or transient populations. In three time-points $(0,670$ and 700 d), 58 different species were identified, 16 of them being retrieved for the first time in human feces. L. rhamnosus, L. ruminis, L. delbrueckii, L. plantarum, $L$. casei and $L$. acidophilus were the most represented, with estimated amounts ranging between 6 and 8 Log (cells g ${ }^{-1}$ ), while the other were detected at 4 or 5 Log (cells $\mathrm{g}^{-1}$ ). 86 Lactobacillus strains belonging to 52 species were identified. 43 seemingly occupied the GIT as true residents, since were detected in a time span of almost 2 years in all the three samples or in 2 samples separated by 670 or $700 \mathrm{~d}$. As a whole, a stable community of lactobacilli

Received 12 February, 2016; accepted 8 March, 2016. *For correspondence. E-mail maddalena.rossi@unimore.it; Tel. 39-0592058589; Fax: 39-059-245156. 'These authors contributed equally to the work. was disclosed, with wide and understudied biodiversity.

\section{Introduction}

The human colon is inhabited by a complex microbial community composed largely of bacteria, whose numbers reaches up to $10^{12}$ cells per gram of intestinal content, belonging to over thousands species (O'Hara and Shanahan, 2006). The host and associated microbiota form an interrelated ecosystem that has major effects on health or diseases. Differences occurring in microbial composition and in metabolic activities deeply influence the health status, since they could promote a healthy homeostasis or could determine the basis for the onset of pathologies (Sekirov et al., 2010). Most of colonic bacteria are members of a resident population that has a long-term association with the host. Other microorganisms found in the colon can inhabit other sections of the gastrointestinal tract (GIT), such as the mouth or the small intestine, shedding alive to the colon but being unable to replicate in this habitat ( $\mathrm{Xu}$ and Gordon, 2003). Furthermore, microorganisms ingested with food and water can transit through the upper intestine and reach alive the hindgut, after being challenged by the low $\mathrm{pH}$ of the stomach, the digestive enzymes, and the toxicity of bile salts. Lactobacilli are quite versatile in terms of ecosystems, and can fall into all these groups.

The genus Lactobacillus includes 217 recognized species enlisted in LPSN database (The List of Prokaryotic Names with Standing in Nomenclature, http://www.bacterio.net, last accessed March 2016) (Parte, 2014). It belongs to the family Lactobacillaceae that, with Enterococcaceae and Streptococcaceae, is included in the order of Lactobacillales within to the gram-positive phylum of Firmicutes. Despite their wide phylogenetic and functional diversity, lactobacilli are invariably saccharolytic, anaerobic or microaerophilic, aciduric or acidophilic, non-sporulating rods (Hammes and Hertel, 2006). Lactobacilli, enterococci and streptococci are included in the functional group of Lactic Acid Bacteria (LAB), since they catabolize hexoses to lactic acid through obligate or facultative homolactic or heterolactic fermentation, the 
latter yielding also two-carbon products (ethanol and/or acetic acid) and $\mathrm{CO}_{2}$.

Lactobacilli inhabit a variety of habitats where carbohydrate-based substrates are available, such as plants, plant-derived matrices, silage, fermented foods (e.g. dairy products, fermented dough, milk, vegetables and meats), spoiled foods, organic matrices and sewage (Hammes and Hertel, 2006). They are also found in the commensal microbiota naturally colonizing diverse districts within the body of humans and animals, such as the oral cavity, the GIT and the vagina (O'Donnell et al., 2013; Douillard and de Vos, 2014). Moreover, humans have safely been ingesting lactobacilli for centuries in fermented foods and beverages, and more recently, in probiotic products. This makes the role of lactobacilli in the ecology of the intestinal microbiota difficult to determine, since they represent a peculiar microbial group, whose population can be made up by transient members or by true inhabitants of diverse districts of the GIT (Walter, 2008).

Despite the positive impact on health of lactobacilli and the use as probiotics, reports providing a detailed description of Lactobacillus population in the human intestinal microbiota, in terms of species and strains, are still rare. Several studies aimed to describe the diversity of Lactobacillus species have been performed through a cultivation step, introducing some bias. Bacteria belonging to the genus Lactobacillus are expected to be cultivable, then easily isolated and enumerated by traditional culture methods (Hartemink and Rombouts, 1999; Van de Casteele et al., 2006). However, unlike lactobacilli in food matrices (e.g. dairy products, meat and vegetables) which can be easily retrieved on selective plates, isolation from human feces is hampered by bifidobacteria, that present similar nutritional and environmental requirements but are much more numerous (Quartieri et al., 2016). Investigations based on cultivation of fecal lactobacilli indicated some recurring species such as $L$. acidophilus, $L$. brevis, $L$. casei, L. crispatus, L. delbrueckii, L. fermentum, L. gasseri, L. jensenii, L. johnsonii, L. mucosae, L. paracasei, L. plantarum, $L$. reuteri, $L$. rhamnosus, $L$. ruminis, $L$. salivarius and L. vaginalis (Reuter, 2001; Dal Bello et al., 2003; Ahrnè et al., 2005; Delgado et al., 2007; Lönnermatk et al., 2012; Öztürk and Meterelliyöz, 2015). Consistently, speciesspecific amplification and sequencing of 16S rRNA gene confirmed the presence of several of the above-cited species (Heilig et al., 2002).

Presently, traditional microbiological methods are overtaken by data provided by next-generation sequencing technologies, such as metagenomic approaches that disclose the relative amounts of the different microorganisms colonizing this habitat (Frank and Pace, 2008). Two different ways are accessible at the moment: $16 \mathrm{~S}$ rRNA gene sequencing and whole genome sequencing
(WGS). 16S rRNA gene sequencing is the most widely exploited for metagenome survey, but in most cases the libraries generated from fecal, colonic or cecal samples of humans fail to retrieve lactobacilli, likely because of their relatively small amounts (Walter, 2008). WGS offers several advantages, such as making available information about the genome of the microorganisms, opening the possibility to analyze their functional capabilities, and providing a picture of the taxonomic composition of microbiota at species- and strain-level, without the bias of $16 \mathrm{~S}$ copy number variation and polymerase chain reaction amplification. In these latter techniques, it is very difficult to reach taxonomic levels of species or strains, whereas shotgun approaches with good coverage could add statistical power to the taxonomical assignments even in low abundances.

In the present study, a mining approach was applied to WGS data already available (Minot et al., 2013), with the aim to longitudinally characterize the fecal lactobacilli of a healthy subject over a two years period.

\section{Results and discussion}

Metagenomic WGS sequences were utilized to explore Lactobacillus biodiversity of human gut microbiota and to discriminate between true inhabitants and transient bacteria. The data, acquired in a previous longitudinal study aimed to explore the variation of the intestinal virome of a male healthy subject (Minot et al., 2013). In our investigation, three time-points were considered and compared $(0,670$ and $700 \mathrm{~d})$ in order evaluate shortterm (between 670 and $700 \mathrm{~d}$ ) and long-term variations (between 0 and 670 or $700 \mathrm{~d}$ ). WGS sequences were analysed using the LMAT-Grand database encompassing complete and partial genome sequences from the NCBI genome database (July 4, 2014), that included genomes from 78 different Lactobacillus species, with 326 strains identified (Ames et al., 2013). Analysis were carried out through the LMAT pipeline, using the Average Read Score to obtain the relative amounts, normalized against the total reads. For more details on the sequence management and the metagenome processing, please refer to Supporting Information Appendix S1.

The relative amounts of the Lactobacillales classifiable in the families of Enterococcaceae Lactobacillaceae and Streptococcaceae were determined. A special attention was paid to the genus Lactobacillus because of the diverse environments that different species can occupy, and for the health promoting properties ascribed to intestinal colonizers. The bacterial concentration of Lactobacillus species and strains was roughly extrapolated from the respective relative amount, assuming that the three fecal samples had the same concentration of microorganisms $\left(1 \times 10^{12}\right.$ cells $\left.g^{-1}\right)$. In order to take in 
account this approximations, only the order of magnitude was considered as reliable and the data were expressed as Log (cells $\mathrm{g}^{-1}$ ). Doing this assumption, major approximations were introduced for the number of cells per $\mathrm{g}$, which is likely different among the three samples. However, the approximation seems reasonable for the aim of comparing the order of magnitude of bacterial abundance, expressed as the Log of the number of cells per $g$ of feces.

\section{Lactobacillales}

WGS led to the identification of a total of $2.110^{7}$ OTUs. At the three time-points, the microbiota was dominated by bacteria, which accounted for the $75 \%, 80 \%$ and $74 \%$ of total microbes at 0,670 and $700 \mathrm{~d}$, respectively. The phyla Firmicutes and Bacteroidetes were always the most represented, whereas the $19 \%-26 \%$ of the bacterial population remained unassigned at taxonomic level (Table 1). Firmicutes accounted for $21 \%-36 \%$ of total bacteria, with the class of Bacilli ranging between $1 \%$ and $3 \%$ of them. The order Lactobacillales accounted for $47 \%, 21 \%$ and $24 \%$ of Bacilli at the three time-points, corresponding to $0.08 \%-0.3 \%$ of the whole bacterial population. The majority of Lactobacillales ( $>92 \%$ ) were ascribable to the families of Streptococcaceae, Lactobacillaceae and Enterococcaceae, in order of abundance (Table 1).

Streptococcaceae were mostly represented by bacteria belonging to the genus Streptococcus (89\%-97\%) that includes both beneficial commensal species, such as $S$. thermophilus, and opportunistic or pathogenic species. Pathogenic species (Krzyściak et al., 2013) of Streptococcus were not identified in the three fecal samples. The species $S$. parasanguinis, S. mitis, S. infantis and $S$. australis which are part of the endogenous microbiota of the oral cavity according to the human oral microbiome database (http://www.homd.org/, last accessed March 2016), accounted at most for the $5 \%$ of streptococci. 59 to 66 Streptococcus species were identified in each sample. Among the health promoting and pro-technological species, S. thermophilus ranged from $5 \%$ to $27 \%$ of streptococci and S. salivarius from $4 \%$ to $11 \%$. Altogether, $S$. thermophilus and $S$. salivarius represented a rather variable percentage of total bacteria $(0.09 \%, 0.005 \%$ and $0.008 \%$ at 0,670 and $700 \mathrm{~d}$ respectively).

The bacteria belonging to the family of Enterococcaceae were mostly represented by the genus Enterococcus $(93 \%-94 \%)$, which included 21 species. E. cecorum, E. faecium and E. faecalis were the most abundant enterococci, ranging from $5 \%$ to $14 \%$, from $8 \%$ to $12 \%$ and from $5 \%$ to $9 \%$ respectively. The species E. gilvus, E. avium, E. columbae, E. pallens, E. moraviensis, E. durans, E. italicus, E. phoeniculicola, E. caccae, E. saccharolyticus, E. dispar and E. haemo-
Table 1. Percentage of bacterial WGS reads attributed to the main phyla and to the class, order, and family of the main intestinal LABs.

\begin{tabular}{lccc}
\hline Phylum/Class/Order/Family & $0 \mathrm{~d}$ & $670 \mathrm{~d}$ & $700 \mathrm{~d}$ \\
\hline Firmicutes & 21 & 26 & 36 \\
$\quad$ Bacilli & 0.8 & 0.5 & 0.7 \\
$\quad$ Lactobacillales & 0.4 & 0.1 & 0.2 \\
$\quad$ Streptococcaceae & 0.3 & 0.05 & 0.08 \\
$\quad$ Lactobacillaceae & 0.04 & 0.03 & 0.03 \\
$\quad$ Enterococcaceae & 0.02 & 0.02 & 0.03 \\
Bacteroidetes & 47 & 52 & 34 \\
Proteobacteria & 6 & 4 & 6 \\
Actinobacteria & 1 & 0.7 & 1 \\
Unassigned bacteria & 21 & 18 & 22 \\
\hline
\end{tabular}

The relative amounts were calculated normalizing the Total Read Score (TRS) of each taxa for the sum of all the TRS of the corresponding sample.

peroxidus were common at the different time points, whereas E. mundtii, E. malodoratus, E. asini and E. villutum were found at both 670 and $700 \mathrm{~d}$, and E. casseliflavus and E. sulfureus only at $700 \mathrm{~d}$.

The genus Lactobacillus covered $94 \%$ to $97 \%$ of Lactobacillaceae, and ranged from $0.03 \%$ to $0.04 \%$ of bacteria. Excluding unassigned biotypes of the family, the other Lactobacillaceae belonged to the genus Pediococcus, phylogenetically intermixed with the genus Lactobacillus, and in particular to the species $P$. claussenii, $P$. acidilactici and $P$. pentosaceus, the two latter being exploited as starters for fermented foods (Leroy and De Vuyst, 2004). Interestingly, also P. claussenii, a common brewery contaminant, was present as a sole biotype in the three samples, being a stable colonizer of the gut of this subject during the 2-year period.

\section{Lactobacillus species}

Bacteria belonging to the genus Lactobacillus ranged from 8.4 to 8.6 Log (cells $\mathrm{g}^{-1}$ ), with a great biodiversity of detected species, being the OTUs spread among 58 different ones out of the 217 recognized. The species ranged from 4 to 8 Log (cells $\mathrm{g}^{-1}$ ). The most abundant ones (L. rhamnosus, L. ruminis, L. delbrueckii, L. plantarum, $L$. casei and $L$. acidophilus; Table 1 ) were the same identified in previous studies utilizing culturebased approaches (Reuter, 2001; Delgado et al., 2007; Wall et al., 2007; Rajilić-Stojanović and de Vos, 2014). Many other, especially among those occurring at 4 or 5 Log (cells $\mathrm{g}^{-1}$ ), emerged for the first time in human fecal samples. This outcome confirmed the drawbacks associated to isolation of fecal lactobacilli occurring in lower amounts by cultural methods (Quartieri et al., 2016).

Most of the taxa identified (e.g. L. animalis, L. acidophilus, L. brevis, L. buchneri, L. casei, L. delbrueckii, $L$. paracasei, $L$. pentosus, $L$. plantarum, $L$. reuteri, $L$. rhamnosus, $L$. rossiae, $L$. ruminis and $L$. sakel) are not 


\begin{tabular}{|c|c|c|c|c|c|c|c|}
\hline Species & O d & $670 \mathrm{~d}$ & $700 \mathrm{~d}$ & Species & $\mathbf{O d}$ & $670 \mathrm{~d}$ & $700 \mathrm{~d}$ \\
\hline L. acidipiscis & 4.7 & 5.2 & 5.5 & L. kisonensis & 5 & 4.8 & - \\
\hline L. acidophilus & 8.1 & 7 & 4.6 & L. mali & 5.7 & 5.1 & 5.5 \\
\hline L. amylolyticus & - & 4.9 & 4.3 & L. mucosae & 6 & 4.8 & 4 \\
\hline L. animalis & - & - & 4.8 & L. murinus & 7.4 & 6.9 & 7.1 \\
\hline L. brevis & 5.8 & 5.3 & 4.4 & L. namurensis & 4.7 & 4.2 & 4.6 \\
\hline L. buchneri & 5.7 & 5.5 & 5.6 & L. oris & 5.8 & 6.2 & 6.3 \\
\hline L. casei & 7.4 & 6.4 & 6.7 & L. otakiensis & 4.9 & 4.7 & - \\
\hline L. ceti & 5.6 & 5.1 & 5 & L. parabrevis & 5.4 & 5.2 & 5.3 \\
\hline L. coleohominis & 4.5 & 4.9 & 5.2 & L. paracasei & 6.1 & 5.6 & 6.3 \\
\hline L. composti & 4.7 & 4.8 & 4.9 & L. parafarraginis & 5.2 & 5.6 & 5.6 \\
\hline L. coryniformis & - & 5.5 & 5.3 & L. pasteurii & 5.6 & - & - \\
\hline L. crispatus & 5.4 & 5 & 5.4 & L. pentosus & 5.7 & 5 & 4.8 \\
\hline L. delbrueckii & 6.6 & 7.2 & 7.4 & L. plantarum & 7.4 & 6.7 & 6.7 \\
\hline L. equi & 5.9 & 5 & 4.9 & L. pobuzihii & 5 & - & - \\
\hline L. equicursoris & - & 4.9 & - & L.psittaci & 5.2 & - & 5.3 \\
\hline L. farciminis & 4.7 & 4.8 & 4.2 & L. reuteri & 6.4 & 5.3 & 5.8 \\
\hline L. farraginis & 5.7 & 5.4 & 4.7 & L. rhamnosus & 8.2 & 7.9 & 8.1 \\
\hline L. fermentum & 7.2 & 5.3 & 5.3 & L. rossiae & 5.2 & 4.4 & 5.4 \\
\hline L. florum & - & 4.7 & 4.7 & L. ruminis & 7.7 & 8 & 7.2 \\
\hline L. fructivorans & 5.5 & 5.3 & 5.2 & L. sakei & 5 & - & - \\
\hline L. gasseri & 5.9 & 5.4 & 4.9 & L. salivarius & 5.9 & 5.9 & 5.9 \\
\hline L. gastricus & 4.9 & - & - & L. sanfranciscensis & 8.6 & 6.1 & 6.5 \\
\hline L. gigeriorum & - & 4.7 & 5.1 & L. shenzhenensis & 5.5 & 5.3 & 5.6 \\
\hline L. harbinensis & 5.4 & 5.5 & 5.4 & L. suebicus & 5.4 & 4.6 & 5.3 \\
\hline L. helveticus & 4.5 & 6.9 & 6.4 & L. ultunensis & 4.5 & 4.7 & - \\
\hline L. hominis & 5.5 & 5.3 & 5.5 & L. vaginalis & 6.1 & 5.9 & 5.9 \\
\hline L. iners & 6.3 & 5.8 & 6.7 & L. versmoldensis & 5.6 & 5.8 & 5.1 \\
\hline L. jensenii & 6.1 & 5.6 & 5.8 & L. vini & 6.2 & - & 5.8 \\
\hline L. johnsonii & 6 & 5.5 & 5.5 & L. zeae & 4.9 & 5.4 & 5.1 \\
\hline
\end{tabular}

Fig. 1. Heatmap of Lactobacillus species in the feces of a healthy subject, at 0,670 and $700 \mathrm{~d}$. Values are Log (cells $\mathrm{g}^{-1}$ ). Colours range from the lowest (deepest green) to the highest (deepest red) abundance. - indicates values falling below the detection limit of $3.9 \mathrm{Log}^{(c e l l s ~} \mathrm{g}^{-1}$ ).

exclusively human commensals, but are found in a variety of other habitats, such as animals, foods, plants, organic spoiled matter or sewage (Hammes and Hertel, 2006). Interestingly, diverse species occurring in plants or foods (e.g. L. acidophilus, L. casei, L. buchneri, L. kisonensis, $L$. parafaraginis and $L$. plantarum), likely ingested with spoiled or fermented aliments, are also enlisted among the bacteria of the human oral microbiome database (http://www.homd.org/). On the other hand, L. composti, $L$. farciminis, $L$. farraginis, $L$. florum, $L$. harbinensis, L. namurensis, L. otakiensis, L. parabrevis, L. pasteurii, $L$. pobuzihii, L. sanfranciscensis, L. shenzhenensis, L. suebicus, $L$. versmoldensis, $L$. vini, $L$. zeae have been described as members of human fecal microbiota for the first time in the present study. These latter species are generally isolated on other organic matrices, such as plants, sewage, foods, organic matter, but have never been associated to humans or animals (Kröckel et al., 2003; Kitahara et al., 2005; Miyamoto et al., 2005; Rodas et al., 2006; Vancanneyt et al., 2006; Endo and Okada, 2007a,b; Scheirlinck et al., 2007; Watanabe et al., 2009; Chen et al., 2010; Endo et al., 2010; Kim et al., 2011; Nam et al., 2011a,b; Cousin et al., 2013; Zou et al., 2013).
Conversely, L. ceti, L. equicursoris, L. gigeriorum, $L$. equi, and $L$. psittaci were found in animal samples, but never in human tissues or specimens (Cousin et al., 2012; Lawson et al., 2001; Morotomi et al., 2002; Vela et al., 2008; Morita et al., 2010). In order to determine which of these species are truly inhabitants of our gut, further insight would be necessary. As a matter of fact, there is always the possibility that the species found in this work could have an external origin as bacteria ingested with food. To address this point, thorough follow-up approaches are necessary and more work has to be done, including efforts in isolating these species or sequencing approaches, to help the evidence of their presence in the GIT as viable bacteria.

Of the 58 Lactobacillus species, 43 were retrieved in the three samples, 9 in two and 6 in one (Fig. 1). Among the species occurring in all the samples, $L$. rhamnosus dominated the Lactobacillus population at all the timepoints $(18.98 \%-56.74 \%)$, being the sole with a mean concentration in the magnitude 8 Log (cells $\mathrm{g}^{-1}$ ). L. ruminis, $L$. murinus, $L$. delbrueckii and $L$. sanfranciscensis were in the magnitude of 7 Log (cells $\mathrm{g}^{-1}$ ) and accounted on average from $4.13 \%$ to $18.96 \%$ of Lactobacillus reads 


\begin{tabular}{|c|c|c|c|c|c|c|c|}
\hline Strain & 0 & $670 \mathrm{~d}$ & $700 \mathrm{~d}$ & Strain & $\mathbf{O d}$ & $670 \mathrm{~d}$ & $700 \mathrm{~d}$ \\
\hline L. acidipiscis KCTC 13900 & 4.7 & 5.2 & 5.5 & L. oris PB013-T2-3 & 5.8 & 6.2 & 6.3 \\
\hline L. amylolyticus DSM 11664 & - & 4.9 & 4.3 & L. otakiensis JCM 15040 & 4.9 & - & - \\
\hline L. animalis DSM 20602 & - & - & 4.8 & L. parabrevis ATCC 53295 & 5.4 & 5.2 & 5.3 \\
\hline L. buchneri CD034 & - & 5.4 & 5 & L. paracasei subsp. paracasei $8700: 2$ & 5.6 & - & - \\
\hline L. buchneri NRRL B-30929 & 5.7 & 4.7 & 5.4 & L. paracasei subsp. paracasei Lpp120 & 5.6 & - & - \\
\hline L. casei 12A & 6.1 & - & 5.8 & L. paracasei subsp. paracasei Lpp122 & - & - & 5.1 \\
\hline L. casei Lc-10 & 5.6 & - & - & L. paracasei subsp. paracasei Lpp126 & - & - & 5.8 \\
\hline L. casei LOCK919 & - & 5.3 & 5.4 & L. paracasei subsp. paracasei Lpp219 & - & 5.2 & - \\
\hline L. casei subsp. casei ATCC 393 & 4.3 & 4.8 & - & L. paracasei subsp. paracasei Lpp229 & - & - & 5.4 \\
\hline L. casei UW4 & - & 4.8 & 5.5 & L. paracasei subsp. paracasei Lpp41 & 5.6 & - & - \\
\hline L. ceti DSM 22408 & 5.2 & 5.1 & 5 & L. paracasei subsp. paracasei Lpp43 & - & - & 5.4 \\
\hline L. coleohominis 101-4-CHN & 4.5 & 4.9 & 5.2 & L. parafarraginis F0439 & 5.2 & 5.6 & 5.6 \\
\hline L. composti JCM 14202 & 4.7 & 4.8 & 4.9 & L. pasteurii CRBIP 24.76 & 5.6 & - & - \\
\hline L. crispatus EM-LC1 & - & - & 5.1 & L. pentosus KCA1 & 5.7 & 5 & 4.8 \\
\hline L. delbrueckii subsp. bulgaricus 2038 & 5.7 & 5.7 & 5.4 & L. plantarum 4_3 & 7.2 & 6.6 & 6.4 \\
\hline L. delbrueckii subsp. bulgaricus ATCC BAA-365 & - & 5.4 & - & L. plantarum AY01 & 5.1 & - & 5 \\
\hline L. delbrueckii subsp. bulgaricus CNCM I-1519 & - & 6.1 & 6 & L. plantarum EGD-AQ4 & 5.2 & - & - \\
\hline L. delbrueckii subsp. bulgaricus CNCM I-1632 & 4.8 & 4.9 & - & L. plantarum subsp. plantarum NC8 & 5.6 & - & - \\
\hline L. delbrueckii subsp. lactis CRL581 & 5.4 & - & - & L. pobuzihii E100301 & 5 & - & - \\
\hline L. equi DPC 6820 & 5.8 & 4.4 & 4.9 & L. psittaci DSM 15354 & 5.2 & - & 5.3 \\
\hline L. equicursoris CIP 110162 & - & 4.9 & - & L. reuteri 15007 & 5.4 & - & - \\
\hline L. farciminis DSM 20184 & 4.7 & 4.8 & 4.2 & L. reuteri Ipuph & - & 4.5 & 4.6 \\
\hline L. farraginis JCM 14108 & 5.7 & 5.4 & 4.7 & L. reuteri mlc3 & 5.6 & - & - \\
\hline L. fermentum 28-3-CHN & 5.4 & - & - & L. rhamnosus 2166 & 6 & - & 5.9 \\
\hline L. fermentum 3872 & 5.8 & 5.3 & - & L. rhamnosus GG & - & - & 5.5 \\
\hline L. fermentum MTCC 8711 & 5.4 & - & - & L. rhamnosus HNO01 & 5.4 & - & - \\
\hline L. fermentum NB-22 & 6 & - & - & L. rhamnosus Lc 705 & 5.6 & - & 5.3 \\
\hline L. fructivorans DSM 20203 & 5.5 & 5.3 & 5.2 & L. rhamnosus LMS2-1 & 6.6 & 5.7 & 6.2 \\
\hline L. gasseri $\mathrm{K7}$ & 5 & 4.8 & 4.5 & L. rhamnosus LOCK908 & 6 & 6.3 & 5.7 \\
\hline L. gigeriorum CRBIP 24.85 & - & 4.5 & 4.6 & L. rossiae DSM 15814 & 5.2 & 4.4 & 5.4 \\
\hline L. harbinensis DSM 16991 & 5.4 & 5.5 & 5.4 & L. ruminis ATCC 25644 & 7.4 & 7.2 & 6.9 \\
\hline L. helveticus CIRM-BIA 953 & - & 4.6 & - & L. ruminis ATCC 27782 & 6.8 & 7.9 & 6.4 \\
\hline L. helveticus MTCC 5463 & - & 5.9 & - & L. ruminis SPM0211 & 6.9 & 6.2 & 6.3 \\
\hline L. hominis CRBIP 24.179 & 5.5 & 5 & 5.4 & L. salivarius ATCC 11741 & - & 4.3 & 5.3 \\
\hline L. iners LEAF 2053A-b & 5.4 & - & - & L. sanfranciscensis TMW 1.1304 & 8.6 & 6.1 & 6.5 \\
\hline L. iners SPIN 2503V10-D & 5.4 & - & - & L. shenzhenensis LY-73 & 5.5 & 5.2 & 5.6 \\
\hline L. jensenii 208-1 & 5.1 & - & - & L. suebicus КстС 3549 & 5.4 & 4.6 & 5.3 \\
\hline L. johnsonii N6.2 & - & 4.6 & - & L. ultunensis DSM 16047 & 4.5 & 4.7 & - \\
\hline L. kisonensis F0435 & 5 & 3.9 & - & L. vaginalis ATCC 49540 & 6.1 & 5.9 & 5.9 \\
\hline L. mali DSM 20444 & 5.7 & 5.1 & 5.5 & L. versmoldensis КСТС 3814 & 5.6 & 5.8 & 5.1 \\
\hline L. mucosae LM1 & 5.9 & 4.8 & 4 & L. vini JP7.8.9 & 5.9 & - & - \\
\hline L. murinus ASF361 & 7.4 & 6.9 & 7.1 & L. zeae КСтС 3804 & 4.9 & 5.4 & 5.1 \\
\hline L. namurensis str. Chizuka 01 & 4.7 & 4.2 & 4.6 & Lactobacillus sp. ASF360 & - & 4.3 & 4.9 \\
\hline
\end{tabular}

Fig. 2. Heatmap of Lactobacillus strains in the feces of a healthy subject, at 0,670 and $700 \mathrm{~d}$. Values are Log (cells $\mathrm{g}^{-1}$ ). Colours range from the lowest (deepest green) to the highest (deepest red) abundance. - indicates values falling below the detection limit of 3.9 Log (cells $\mathrm{g}^{-1}$ ).

over the three samples. L. ruminis, L. delbrueckii, L. sanfranciscensis and $L$. acidophilus, identified at all the timepoints, presented wide quantitative differences among the samples and were particularly abundant at a sole timepoint where peaked up to 8 Log (cells $\mathrm{g}^{-1}$ ). The vast majority of the recurrent species (27) showed a mean concentration laying in the magnitude of 5 Log (cells $\mathrm{g}^{-1}$ ), and generally accounted for $<1 \%$ of Lactobacillus reads. Seven species (L. rossiae, L. mucosae, L. coleohominis, L. pentosus, L. composti, L. farciminis, L. namurensis) were present at concentration in the magnitude of 4 Log (cells $\mathrm{g}^{-1}$ ), accounting for $<0.1 \%$. For the 43 species occurring at all the time-points, no correlation was observed between the mean abundance and the variation range over the three samples (Supporting Information Fig. S1A).

Most of the persistent species (33 out of 43), including L. delbrueckii, L. murinus, L. rhamnosus and L. ruminis, and also the majority of the less abundant species with mLog (cells $\mathrm{g}^{-1}$ ) $<6$, exhibited a relevant quantitative stability, with 1 or less magnitudes of difference among the time-points, regardless of their abundance. Only for L. helveticus, L. sanfranciscensis and $L$. acidophilus $\Delta$ Log (cells $\mathrm{g}^{-1}$ ) was higher than 2, with the latter showing the widest range (3.6 magnitudes) of concentrations over the three time point. Concentration variation over short and long periods was also considered (Supporting Information Fig. S1B), the former being the variation between 670 and $700 \mathrm{~d}$, and the latter as the variation between $0 \mathrm{~d}$ and the mean of the two last time points. The vast majority of the species differed by less than 1 magnitude order over both the short and long periods. Only $L$. acidophilus presented short-term and long-term variation both higher than $2 \Delta$ Log (cells ${ }^{-1}$ ), whereas $L$. fermentum, $L$. helveticus, $L$. mucosae and $L$. sanfranciscensis showed high long-term variation, but remained stable over the short period. 


\section{Lactobacillus strains}

WGS data allowed a deeper investigation of bacteria identified, making possible to trace 86 Lactobacillus spp. strains in one or more samples. The lactobacilli identified at strain level were the $58.30 \%, 48.67 \%$ and $21.86 \%$ of total Lactobacillus OTUs, at the three timepoints. They belonged to 52 species, the following ones presenting more than one biotype: L. paracasei (8 biotypes), L. rhamnosus (5), L. casei and L. delbrueckii (5), $L$. fermentum and $L$. plantarum (4), L. ruminis (3), $L$. buchneri, $L$. helveticus and $L$. iners (2). A positive relationship was generally observed between the number of biotypes and the abundance of each species (data not shown). On the other hand, 8 biotypes of $L$. paracasei accounted, on average, for $0.41 \%$ of total lactobacilli, whereas $L$. sanfranciscensis and $L$. murinus were represented by a sole biotype, although they results among the most abundant Lactobacillus species.

Among the 86 Lactobacillus biotypes, 43 strains seemingly occupied the GIT as true residents: 34 occurred in all the three samples, and further 9 were identified both at $0 \mathrm{~d}$ and at one of the remainder samples, 670 or $700 \mathrm{~d}$ (Fig. 2). Taking into account the recurrent presence of the same strains over more than 22 months, albeit the availability of a small set of data (only 3 points), these 43 strains could be considered as permanent colonizers of the GIT. It should not be excluded that quantitative fluctuations, responsible of concentrations not emerging above the limit of detection of 3.9 Log (cells $\mathrm{g}^{-1}$ ), may have hindered the identification of low abundance colonizers, such as 9 strains detected at 670 and $700 \mathrm{~d}$, at magnitudes of 4 or 5 Log (cells $\mathrm{g}^{-1}$ ), but not found at $0 \mathrm{~d}$. Some of the permanent colonizers belong to species generally associated to human gut microbiota (e.g. L. casei, L. crispatus, L. delbrueckii, L. fermentum, L. gasseri, L. johnsonii, L. paracasei, L. plantarum, L. reuteri, L. rhamnosus, L. ruminis, $L$. salivarius and $L$. vaginalis), while many others belong to species that have never been previously associated to humans ( $L$. composti, $L$. farciminis, $L$. farraginis, $L$. harbinensis, L. namurensis, L. parabrevis, L. sanfranciscensis, L. shenzhenensis, L. suebicus, L. versmoldensis, L. zeae), thus modifying the vision of Lactobacillus ecology. It is impossible to determine whether these bacteria inhabit the colon and fill the functional niches of this ecosystem, or are members of a bacterial population residing in another part of the GIT and shedding to the hindgut. However, it seems implausible that these strains are transient bacteria, occasionally ingested twice over a period of 2 years.

Most of the persistent strains (30 out of 34) showed a relevant quantitative stability, with 1 or less magnitudes of difference among the time-points, regardless of their mean concentration, and over both the short and the long period (Supporting Information Fig. S1C and D). The exceptions were L. equi DPC 6820, L. mucosae LM1, and L. sanfranciscensis TMW 1.1304, which presented quantitative fluctuations $>1$ Log (cells $\mathrm{g}^{-1}$ ) over the long time span, and L. ruminis ATCC 27782 which presented quantitative fluctuations $>1$ Log (cells $\mathrm{g}^{-1}$ ) over both the long and the short period.

\section{Conclusions}

Up to now, human GIT lactobacilli were perceived not only as a marginal population, but also as one of the major transient components of microbiota, originating from exogenous sources (Walter, 2008). Despite the limit of a longitudinal approach on a single subject, the results herein presented indicate the presence of a stable community of lactobacilli, with wide and understudied biodiversity. The low concentration of most of the species suggests a sub-dominant role in the colonic ecosystem. With this new insight, novel questions arise. A major challenge is determining the specific GIT district where this plethora of Lactobacillus species replicates and grows, in order to discriminate if they are indigenous resident of the colon, or whether they reach it shedding from upstream sites that they colonize. This can make the difference in terms of microbe-immune system relationship, since in the latter case lactobacilli can actively interact with GALT (gut-associated lymphoid tissue) exerting relevant immunomodulatory properties. Moreover, a recent study on the interaction between gut microbiome and virome highlighted the role of phages and prophages in modulating the bacterial structure and function of the bacterial community, with lytic lifestyles being effective in determining the dynamics of subdominant bacteria (Minot et al., 2013; Ogilvie and Jones, 2015). Bacteriophages infecting lactobacilli are numerous, but the knowledge of their biology is still limited to the industrially relevant ones, and their role in shaping the community of intestinal Lactobacillus population is not known so far (Mahony and van Sinderen, 2014). As a whole, lactobacilli resulted a stable, relatively abundant, and very biodiverse community within the gut microbiota, but the current status of knowledge on colonic lactobacilli remains a major challenge.

\section{Acknowledgements}

The study was funded by Project SAF2012-31187 from MINECO (Ministerio de Economía y Competitividad, Madrid, Spain), PROMETEOII/2014/065 from Generalitat Valenciana (Valencia, Spain), and SIME2013.0627 (Fondazione Cassa di Risparmio di Modena, Italy). 


\section{References}

Ames, S.K., Hysom, D.A., Gardner, S.N., Lloyd, G.S., Gokhale, M.B., and Allen, J.E. (2013) Scalable metagenomic taxonomy classification using a reference genome database. Bioinformatics 29: 2253-2260.

Ahrné, S., Lönnermark, E., Wold, A.E., Aberg, N., Hesselmar, B., Saalman, R., et al. (2005) Lactobacilli in the intestinal microbiota of Swedish infants. Microbes Infect 7: 1256-1262.

Chen, Y.S., Miyashita, M., Suzuki, K., Sato, H., Hsu, J.S., and Yanagida F. (2010) Lactobacillus pobuzihii sp. nov., isolated from pobuzihi (fermented cummingcordia). Int $J$ Syst Evol Microbiol 60: 1914-1917.

Cousin, S., Gulat-Okalla, M.L., Motreff, L., Gouyette, C., Bouchier, C., Clermont, D., and Bizet, C. (2012) Lactobacillus gigeriorum sp. nov., isolated from chicken crop. Int J Syst Evol Microbiol 62: 330-334.

Cousin, S., Motreff, L., Gulat-Okalla, M.L., Gouyette, C., Spröer, C., Schumann, P., et al. (2013) Lactobacillus pasteurii sp. nov. and Lactobacillus hominis sp. nov. Int $J$ Syst Evol Microbiol 63: 53-59.

Dal Bello, F., Walter, J., Hammes, W.P., and Hertel, C. (2003) Increased complexity of the species composition of lactic acid bacteria in human feces revealed by alternative incubation condition. Microb Ecol 45: 455-463.

Delgado, S., Suárez, A., and Mayo, B. (2007) Dominant cultivable Lactobacillus species from the feces of healthy adults in northern Spain. Int Microbiol 10: 141145.

Douillard, F.P., and de Vos, W.M. (2014) Functional genomics of lactic acid bacteria: from food to health. Microb Cell Fact 13: S8.

Endo, A., and Okada, S. (2007a) Lactobacillus composti sp. nov., a lactic acid bacterium isolated from a compost of distilled shochu residue. Int $J$ Syst Evol Microbiol 57: 870-872.

Endo, A., and Okada, S. (2007b) Lactobacillus farraginis sp. nov. and Lactobacillus parafarraginis sp. nov., heterofermentative lactobacilli isolated from a compost of distilled shochu residue. Int J Syst Evol Microbiol 57: 708-712.

Endo, A., Futagawa-Endo, Y., Sakamoto, M., Kitahara, M., and Dicks, L.M. (2010) Lactobacillus florum sp. nov., a fructophilic species isolated from flowers. Int $J$ Syst Evol Microbiol 60: 2478-2482.

Frank, D.N., and Pace N.R. (2008) Gastrointestinal microbiology enters the metagenomics era. Curr Opin Gastroenterol 24: 4-10.

Hammes, W.P., and Hertel, C. (2006) The genera Lactobacillus and Carnobacterium. In The Prokaryotes, 3rd edn, Vol. 4. Dworkin, M., Falkow, S., Rosenberg, E., Schleifer, K.H., and Stackenbrandt, E. (eds). New York: Springer, pp. 319-402.

Hartemink, R., and Rombouts, F.M. (1999) Comparison of media for the detection of bifidobacteria, lactobacilli and total anaerobes from faecal samples. J Microbiol Methods 36: 181-192.

Heilig, H.G., Zoetendal, E.G., Vaughan, E.E., Marteau, P., Akkermans, A.D., and de Vos, W.M. (2002) Molecular diversity of Lactobacillus spp. and other lactic acid bacte- ria in the human intestine as determined by specific amplification of $16 \mathrm{~S}$ ribosomal DNA. Appl Environ Microbiol 68: 114-123.

Kim, D.W., Choi, S.H., Kang, A., Nam, S.H., Kim, D.S., Kim, R.N., et al. (2011) Draft genome sequence of Lactobacillus zeae KCTC 3804. J Bacteriol 193: 5023.

Kitahara, M., Sakata, S., and Benno, Y. (2005) Biodiversity of Lactobacillus sanfranciscensis strains isolated from five sourdoughs. Lett Appl Microbiol 40: 353-357.

Kröckel, L., Schillinger, U., Franz, C.M., Bantleon, A., and Ludwig, W. (2003) Lactobacillus versmoldensis sp. nov., isolated from raw fermented sausage. Int J Syst Evol Microbiol 53: 513-517.

Krzyściak, W., Pluskwa, K.K., Jurczak, A., and Kościelniak, D. (2013) The pathogenicity of the Streptococcus genus. Eur J Clin Microbiol Infect Dis 32: 1361-1376.

Lawson, P.A., Wacher, C., Hansson, I., Falsen, E., and Collins, M.D. (2001) Lactobacillus psittaci sp. nov., isolated from a hyacinth macaw (Anodorhynchus hyacinthinus). Int J Syst Evol Microbiol 51: 967-970.

Leroy, F., and De Vuyst, L. (2004) Lactic acid bacteria as functional starter cultures for the food fermentation industry. Trends Food Sci Technol 15: 67-78.

Lönnermark, E., Nowrouzinan, F., Adlerberth, I., Ahrné, S., Wold, A., and Friman, V. (2012) Oral and faecal lactobacilli and their expression of mannose-specific adhesins in individuals with and without $\lg \mathrm{A}$ deficiency. Int $\mathrm{J}$ Med Microbiol 302: 53-60.

Mahony, J., and van Sinderen, D. (2014) Current taxonomy of phages infecting lactic acid bacteria. Front Microbio/ 5: 7.

Minot, S., Bryson, A., Chehoud, C., Wu, G.D., Lewis, J.D., and Bushman, F.D. (2013) Rapid evolution of the human gut virome. Proc Natl Acad Sci USA 110: 12450-12455.

Miyamoto, M., Seto, Y., Hao, D.H., Teshima, T., Sun, Y.B., Kabuki, T., et al. (2005) Lactobacillus harbinensis sp. nov., consisted of strains isolated from traditional fermented vegetables 'Suan cai' in Harbin, Northeastern China and Lactobacillus perolens DSM 12745. Syst Appl Microbiol 28: 688-694.

Morita, H., Shimazu, M., Shiono, H., Toh, H., Nakajima, F., Akita, H., et al. (2010) Lactobacillus equicursoris sp. nov., isolated from the faeces of a thoroughbred racehorse. Int J Syst Evol Microbiol 60: 109-112.

Morotomi, M., Yuki, N., Kado, Y., Kushiro, A., Shimazaki, T., Watanabe, K., and Yuyama T. (2002) Lactobacillus equi sp. nov., a predominant intestinal Lactobacillus species of the horse isolated from faeces of healthy horses. Int $J$ Syst Evol Microbiol 52: 211-214.

Nam, S.H., Choi, S.H., Kang, A., Kim, D.W., Kim, R.N., Kim, A., et al. (2011a) Genome sequence of LactobacilIus farciminis KCTC 3681. J Bacteriol 193: 1790-1791.

Nam, S.H., Choi, S.H., Kang, A., Kim, D.W., Kim, R.N., Kim, D.S., et al. (2011b) Genome sequence of Lactobacillus suebicus KCTC 3549. J Bacteriol 193: 5532-5533.

O'Donnell, M.M., O'Toole, P.W., and Ross, R.P. (2013) Catabolic flexibility of mammalian-associated lactobacilli. Microb Cell Fact 12: 48.

O'Hara, A.M., and Shanahan, F. (2006) The gut flora as a forgotten organ. EMBO Rep 7: 688-693.

Ogilvie, L.A., and Jones, B.V. (2015) The human gut virome: a multifaceted majority. Front Microbiol 6: 918. 
Öztürk, M., and Meterelliyöz, M. (2015) Practical identification of human originated Lactobacillus species by amplified ribosomal DNA restriction analysis (ARDRA) for probiotic use. Mol Biol Rep 42: 1323-1332.

Parte, A.C. (2014) LPSN-list of prokaryotic names with standing in nomenclature. Nucleic Acids Res 42: D613-D616.

Quartieri, A., Simone, M., Gozzoli, C., Popovic, M., D’Auria, G., Amaretti, A., et al. (2016) Comparison of culturedependent and independent approaches to characterize fecal bifidobacteria and lactobacilli. Anaerobe, 38: 130137.

Rajilić-Stojanović, M., and de Vos, W.M. (2014) The first 1000 cultured species of the human gastrointestinal microbiota. FEMS Microbiol Rev 38: 996-1047.

Reuter, G. (2001) The Lactobacillus and Bifidobacterium microflora of the human intestine: composition and succession. Curr Issues Intest Microbiol 2: 43-53.

Rodas, A.M., Chenoll, E., Macián, M.C., Ferrer, S., Pardo, I., and Aznar, R. (2006) Lactobacillus vini sp. nov., a wine lactic acid bacterium homofermentative for pentoses. Int J Syst Evol Microbiol 56: 513-517.

Scheirlinck, I., Van der Meulen, R., Van Schoor, A., Cleenwerck, I., Huys, G., Vandamme, P., et al. (2007) Lactobacillus namurensis sp. nov., isolated from a traditional Belgian sourdough. Int J Syst Evol Microbiol 57: 223-227.

Sekirov, I., Russell, S.L., Antunes, L.C., and Finlay, B.B. (2010) Gut microbiota in health and disease. Physiol Rev 90: 859-904.

Vancanneyt, M., Naser, S.M., Engelbeen, K., De Wachter, M., Van der Meulen, R., Cleenwerck, I., et al. (2006) Reclassification of Lactobacillus brevis strains LMG 11494 and LMG 11984 as Lactobacillus parabrevis sp. nov. Int J Syst Evol Microbiol 56: 1553-1557.

Van de Casteele, S., Vanheuverzwijn, T., Ruyssen, T., Van Assche, P., Swings, J., and Huys, G. (2006) Evaluation of culture media for selective enumeration of probiotic strains of lactobacilli and bifidobacteria in combination with yoghurt or cheese starters. Int Dairy J 16: 1470-1476.

Vela, A.I., Fernandez, A., Espinosa de los Monteros, A., Goyache, J., Herraez, P., Tames, B., et al. (2008) Lactobacillus ceti sp. nov., isolated from beaked whales
(Ziphius cavirostris). Int J Syst Evol Microbiol 58: 891894.

Wall, R., Fitzgerald, G., Hussey, S., Ryan, T., Murphy, B., Ross, P., and Stanton, C. (2007) Genomic diversity of cultivable Lactobacillus populations residing in the neonatal and adult gastrointestinal tract. FEMS Microbiol Ecol 59: 127-137.

Walter, J. (2008) Ecological role of lactobacilli in the gastrointestinal tract: implications for fundamental and biomedical research. Appl Environ Microbiol 74: 49859496.

Watanabe, K., Fujimoto, J., Tomii, Y., Sasamoto, M., Makino, H., Kudo, Y., and Okada, S. (2009) Lactobacillus kisonensis sp. nov., Lactobacillus otakiensis sp. nov., Lactobacillus rapi sp. nov. and Lactobacillus sunkii sp. nov., heterofermentative species isolated from sunki, a traditional Japanese pickle. Int J Syst Evol Microbiol 59: 754-760.

Xu, J., and Gordon, J.I. (2003) Honor thy symbionts. Proc Natl Acad Sci USA 100: 10452-10459.

Zou, Y., Liu, F., Fang, C., Wan, D., Yang, R., Su, Q., et al. (2013) Lactobacillus shenzhenensis sp. nov., isolated from a fermented dairy beverage. Int $J$ Syst Evol Microbiol 63: 1817-1823.

\section{Supporting Information}

Additional Supporting Information may be found in the online version of this article at the publisher's web-site:

Fig. S1. Panel A and C: variation of the amounts of Lactobacillus species $(A)$ and strains $(C)$ within the three timepoints, expressed as $\Delta$ Log (cells $\mathrm{g}^{-1}$ ), plotted against their mean abundance, expressed as $\mathrm{mLog}$ (cells $\mathrm{g}^{-1}$ ). Panel $\mathrm{B}$ and D: long term variation of the amounts of Lactobacillus species (B) and strains (D), plotted against their respective short term variation. Short term variation is calculated as the variation between $\log$ (cells $\mathrm{g}^{-1}$ ) at 670 and $700 \mathrm{~d}$, long term variation as the variation between Log (cells $\mathrm{g}^{-1}$ ) at $0 \mathrm{~d}$ and the mean of the two last time points.

Experimental procedure. 